\title{
A Performance Evaluation of WLAN-Femtocell-LTE beyond the Capacity Crunch. Does Femtocell have to overcome WLAN or can they coexist in HetNets?
}

\author{
K.C.Silva, J.P.L.Araújo, C.R.L.Francês, \\ Federal University of Pará, Computer and Telecommunications Engineering Faculty, Belém, Pará, Brazil, \\ Email: \{ketyllen, jasmine, rfrances\}@ufpa.br
}

\begin{abstract}
This paper originates from a practical experiment based on Mesh Networks. The promising results obtained from this experiment prompted us to discuss in greater depth whether Wireless Local Area Networks (WLAN) are still useful for current communications. This means that on the one hand femtocells can be defined as small base stations that use the same frequency bands as cellular networks. This has become a key feature in LTE to improve capacity and enhance data rates. On the other hand, WLAN, which in terms of infrastructure is similar to femtocells due to the IP backhaul network, seems to have outgrown its usefulness largely because it is being replaced by femtocell technology. In this paper, an attempt is made to clarify this uncertainty through an extensive analysis beyond the capacity crunch of both technologies. The work was undertaken by first working separately and in a second stage, working together to provide possible hybrid access to help offload traffic. Using cellular systems to underpin our research, we give examples of the capacity crunch for femtocells and WLAN through an extensive simulation campaign.
\end{abstract}

Index Terms - Femtocells, LTE, WLAN, Performance Evaluation.

\section{INTRODUCTION}

Long Term Evolution (LTE) is considered one of the most important cellular systems that has been put forward by 3rd Generation Partnership Project (3GPP) in Release 8. It aims to provide higher data rates for future mobile applications [1]. LTE adopts Orthogonal Frequency Division Multiple Access (OFDMA) as the base technique for resource sharing among multiple users. 3GPP further extended the original proposal of LTE, which is known as LTE-Advanced. This proposal aims to achieve data rates up to 1 Gbps and 500 Mbps in downlink and uplink, respectively. Indeed, 3GPP considers deployment of Femtocell technology as potential extensions of LTE-Advanced to expand the coverage and capacity.

The demand for indoor wireless multimedia and ongoing trends of mobile convergence is paving the way for the installation of the femtocell industry. Femtocells may be open access or closed access. Open access allows an arbitrary user to use the femtocell, whereas in closed access, the use is restricted to users that are explicitly approved by the owner. While the ultimate goal of femtocell is to improve the efficiency, coverage and services at a reduced cost of operation, the possibility of arbitrary handovers between the existing eNodeB (enhanced NodeB) and HeNB (Home eNodeB), poses new challenges [2].

On the other hand, the WLANs were designed as an extension of the terrestrial Local Area Networks (LANs), to provide network connectivity with restricted mobility. While cellular networks consist of a 
dedicated terrestrial backbone, WLANs generally connect directly to the IP networks through Digital Subscriber Loop (DSL) or the Ethernet backbone network [3]. In 5G networks, one prominent feature is the prevalence of small cells for high capacity and an energy-efficient form of service provision. The old concept of cells is disrupted and replaced by the concept of multiple-layer heterogeneous cells.

A Heterogeneous Network (HetNet) consists of a series of low-power nodes that are distributed throughout the existing macro cell network. The small cells transmit at significantly lower power levels. This deployment leads to an improvement in the spectral efficiency per unit area, as the low-power cells make it possible to remove coverage holes in the macro-only network and increase the capacity in zones with a very high traffic volume. This means that Heterogeneous Networks can be viewed as an improvement in the major performance enablers of LTE-Advanced.

As mobile data traffic is rapidly growing, it is predicted that in the next decade, an estimated 1000 - fold increase in capacity could be achieved as a result of the staggering growth of mobile data traffic [4]. In summary, as smartphones continue to proliferate, there is a clear and urgent need to improve the efficiency and capacity of broadband networks. Equally clearly, however, no single approach will solve the huge problem caused by the exponential growth in network traffic. On the other hand, there is a need to reduce operational expenditure.

The most important outcome of an optimized network is a satisfied end user. As the improvements in network performance allow the usage of more and more time and bandwidth, as well as critical applications such as Voice over IP (VoIP) and streaming, it is important to ensure that the mobile situations do not degrade the quality of these services.

One of the most promising solutions to cope with the sharp rise in mobile data traffic is mobile data offloading, which refers to the use of supplementary network technologies and innovative techniques to offload data traffic from cellular networks and thereby alleviate their congestion [5].

The Mobile data offload is one of the implementations that uses small cell technologies like Wi-Fi to provide data services to cellular users in a more efficient and economically viable manner. Other small cell technologies like femtocells may also be employed for the same purpose but Wi-Fi is attracting more attention from the cellular industry to cater to the rising demands for data by the users.

It is estimated that $2 / 3$ of calls and over $90 \%$ of data traffic in a cellular network, occur in an indoor environment. Some research has shown that $45 \%$ of households and $45 \%$ of companies have a bad experience regarding indoor coverage [6]. Providing good indoor coverage for consumers has become a major challenge for operators, as it does not simply involve offering a good voice service, but also high data transfers and video.

\section{StATEMENT OF THE PROBlem}

Owing to the problem of penetration losses, the indoor user requires high power from the serving Base Station (BS), which means other users have less power and as a result, the overall system throughput is reduced. It is also very expensive to have a large enough number of outdoor BSs to meet the needs of a high 
capacity network. The large number of BSs impose a larger burden on network planning and optimization as well. The modulation and coding schemes for high data rates used in the standards mentioned above, require good channel conditions, which means that in the case of indoor coverage, Quality of Service (QoS) cannot be guaranteed, due to variations in channel conditions [7].

Doubts about the viability and effectiveness of the use of WLANs to enable a cellular network to offload traffic, have been the subject of research and uncertainty has naturally arisen. On the other hand, femtocells that are structurally similar to cellular networks in terms of their frequency band and physical layer are currently being regarded as a panacea and this has led to a growing sense that the use of WLAN is declining; it will probably not be used anymore and be replaced by femtocells. But the question is, is this true?

To address this issue, this paper examines the effect of deploying Femtocell and WLAN to offload traffic from the LTE system and in a further investigation, implements both as offload traffic. In other words, LTEbased simulations in a comprehensive system were used to study the impact of femtocells and WLAN deployment until the capacity crunch of the system performance. In addition, the investigation aims to analyze the conditions required for the use of WLAN today as an alternative way of offloading traffic from LTE networks, either alone or with femtocells.

The objective is not to exhaust the subject or provide definitive results, but show some important counter arguments that can either qualify or contribute to the discussion. This paper seeks to provide some insights into the evolving course of communication technology with the aim of exploring the use of femtocells and WLANs and evaluating the correlation between the indicators of QoS and Quality of Experience (QoE). The study also examines the effectiveness of using LTE femtocells/WLANs and especially what effects, in terms of benefits and drawbacks, might result from this combination of technologies.

\section{A GENERAL SURVEY OF RELATED WORK}

Some recent papers have focused on the question of offloading cellular traffic in WLAN networks. In [8] the authors carry out a quantitative study of the performance of $3 \mathrm{G}$ mobile data offloading through $\mathrm{WiFi}$ networks. They recruited about 100 iPhone users from metropolitan areas and collected statistics on their WiFi connectivity during a period of about two and half weeks. Their findings led them to conclude that offloading this traffic collected is an effective means of accommodating both the current and future growth in traffic. The main difference between this proposed work and [8] is the capacity crunch which is analysed to determine the performance of both WiFi, and Femtocells.

In [9] the authors proposed and evaluated an integrated architecture which involved exploiting the opportunistic networking paradigm to migrate data traffic from cellular networks to metropolitan WiFi access points (APs). In quantifying the benefits of deploying this kind of architecture, they took account of the case of bulk file transfer and video streaming over $3 \mathrm{G}$ networks and simulated delivery data by means of a real mobility data set of 500 taxis in an urban area. They attempted to quantitatively evaluate the benefits of citywide WiFi offloading using large- scale real traces. Again, the main difference between our proposal and theirs is the fact that we simulated until beyond the capacity crunch and analysed the femtocells as well. 
In [10] the authors are concerned with vehicular Internet access in three different cities, (a particularly challenging question for mobile connectivity). They found that on average $3 \mathrm{G}$ access is available $87 \%$ of the time, while WiFi access (through open APs) is available only $11 \%$ of the time. Thus, they employed techniques to combine multiple interfaces (that incurred different costs) with ubiquitousness, with the goal of reducing the total cost of data transfer while meeting application requirements. They applied these techniques to the context of augmenting $3 \mathrm{G}$ with WiFi in mobile environments.

In [11] the authors proposed a dynamic system to offload traffic between LTE and WLAN.

In [12] the authors discuss the issues and solutions related to enhancing performance when offloading traffic in LTE-A. However, unlike this work, they did not make simulations or carry out practical experiments.

The most recent paper about this subject is [13]. In [13] there is a review of mobile data offloading from LTE to Wifi access networks. The authors also describe various offload strategies and compare them in different scenarios. The main difference between this work and [13] is the simulation that is widely employed to prove whether or not it is still feasible to use the Wifi as an alternative to offload the traffic from LTE Networks efficiently.

\section{THEORETICAL BACKGROUND}

A. LTE

In an attempt to find solutions which can make data transmission more efficient, and against a background where there is a significant rise in the volume of this traffic, the LTE standard was put forward as the next stage to be followed - leading to the $4 \mathrm{G}$ mobile system and preceded by the $2 \mathrm{G}$ and $3 \mathrm{G}$ networks. It is expected that its development will give rise to an improvement in performance as well as reducing the total bit cost, and that this will bring about a wider dissemination of mobile services. 3GPP is responsible for its standardization. [14]

The first release of LTE was published in March 2009 and is referred to as LTE Rel-8. Compared with 3G technologies, such as 3GPP HSPA,1 LTE Rel-8 offers higher peak data rates due to larger system bandwidth (up to $20 \mathrm{MHz}$ was allowed) and higher-order multiple-input multiple-output (MIMO) spatial processing techniques.

In LTE networks there is a new architecture, which is completely different from what has been used in previous technologies, and an example of this is the base station, called eNodeB where the LTE carries out processing tasks that were previously undertaken by the RNC (Radio Network Controller). It should be noted that the eNodeB will also be responsible for handover decisions through communication between the elements by means of the X2 interface. However, it is possible that the lack of communication with X2 (optional interface), means that communication between the eNodeB will be accomplished through other channels via the Access Gateway. [2] 


\section{B. Femtocell}

The concept of femtocell forms a part of the attempt by the telecommunication industry to provide communication of a high performance, with high-quality services for home users. It is usually installed indoors and connected to the user's broadband service modem much like a WiFi access point. Femtocells provide a high-speed data connection to subscribers within a small range [16].

Femtocells are small base stations with the same functionality as macrocells, but they only have the power to serve a restricted environment (10-30 meters), are low cost, can only cater for a small number of users and are installed by the users themselves. One factor that should be taken into consideration is that the decision about how to install these femtocells is up to the user. In other words, it involves a plug and play device, and requires little planning, since it only relies on the skills of built-in self-configuration to minimize the impact on the macrocells through self- provisioning parameters.

Fig. 1 illustrates the overall LTE network architecture in the presence of HeNBs. It should be pointed out that the Rel.9 LTE femtocells are not interconnected with the standard X2 interface, as in the case of eNB, while the deployment of a HeNB GW is not mandatory.

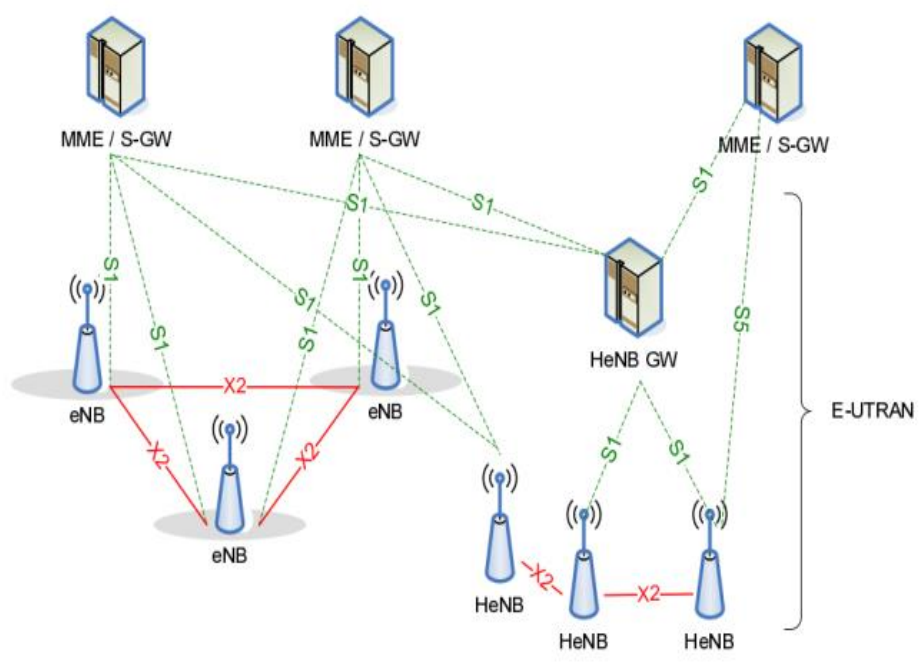

Fig.1. Overall E-UTRAN Architecture with deployed HeNB GW [15].

Both the femtocells and Wifi access point have similarities but have difference as well, both uses internet as a backhaul network and thus the QoS mainly depends on the backhaul. However, the femtocell implements cellular technology while Wi- Fi are WLANs and mainly used for data services. Table 1 shows the main difference between femtocells and Wifi. [3]

TABle I. Femtocell AND Wifi SPECIFICATIONS

\begin{tabular}{ccc}
\hline Specifications & Femtocell & Wifi \\
\hline Data Rates & $7.2-14.4 \mathrm{Mbps}$ & 11 and $54 \mathrm{Mbps}$ \\
Operational Frequency & $1.9-2.6 \mathrm{Ghz}$ & $2.4-5 \mathrm{Ghz}$ \\
Power & $10-100 \mathrm{~mW}$ & $100-200 \mathrm{~mW}$ \\
Range & $10-30 \mathrm{~m}$ & $100-200 \mathrm{~m}$ \\
Services & Primarily Voice and Data & Primarily Data and Voice \\
\hline
\end{tabular}


The essential difference between a femtocell and a macrocell is in their respective backhauls. A femtocell backhaul is an interface for the Mobile Core Network (MCN) through the public Internet, as opposed to the backhaul of a macrocell, which has dedicated lines to the MCN. While it usually takes less than $100 \mathrm{~ms}$ for a handover between macrocells, it can take well over $200 \mathrm{~ms}$ to transmit a single message in the public Internet.

Currently, there is no standardized procedure that specifically handles handovers involving femtocells. However, if the legacy handover procedure were to be applied to these handovers, the introduction of the public Internet between the HeNB and the MCN would introduce additional latency. [18]

Moreover, owing to the small size of the femtocells, the frequency of handovers will also increase. As a result, a fast moving UE may find it hard to remain connected with fast moving femtocells in its path. [18] Hence, the concept of a small, low-cost, customer-deployable home base station offers the prospect of reducing churn by improving indoor coverage and saving costs by offloading the macro radio network.

However, as the initial femtocell service launches are completed and the results fed back into the market, it is clear there are a number of technical standards, business case studies and regulatory challenges that still need to be addressed if femtocells are to really take off in the consumer market.

\section{WLAN}

Wireless LAN has come a long way since its inception in the early nineties. After a decade of proprietary products, wireless LAN suddenly became a mass market with the adoption of the $802.11 \mathrm{~b}$ standard and the formation of Wifi. Since then there has been a continuous stream of innovations in products and standards.

The IEEE 802.11 wireless local area network is one of the most widely used wireless access networks. A wide range of devices, such as laptops, mobile phones, and sensors, are equipped with WLAN interfaces. Current Wifi systems based on IEEE 802.11a/g support a peak physical layer data rate of 54 Mbps and generally provide indoor and outdoor coverage of over a few thousand square meters, which makes them suitable for business enterprise networks and public hot spot scenarios such as airports and hotels [19]. The 802.11 Lan is based on a cellular architecture where the system is subdivided into cells called Basic Service Set (BSS).

According to the Wifi Alliance, about 200 million households use Wifi networks, and there are about 750,000 Wifi hotspots worldwide. Wifi is used by over 700 million people and there are about 800 million new Wifi devices every year. [20] In Brazil the number of Wifi hotspots grew 189\% from 2013 to 2014. Thus, it now ranks eighth in the ranking of countries with greater availability to Wifi. Most operators around the world (e.g., China Mobile, AT\&T) are aggressively deploying WLANs for additional capacity since WiFi is cheap and easy to deploy at scale [21]-[23].

Operators are thus under pressure to cope with this amount of traffic, and find realistic solutions at a reasonable cost, as well as a way to improve data throughput by including additional access technologies which already exist. WLAN is a promising candidate for this kind of solution, because there are a huge number of networks already rolled out worldwide and the end user devices are very price competitive. 
WLAN is already integrated in most of the current smartphones. However, in most devices in the market today, WLAN and 3GPP technologies can be regarded as two separate devices in one box: Specific IP flows are routed over the WLAN access without traversing the 3GPP nodes. A first architecture for the integration of WLAN networks in 3GPP was defined by Release 6, as the Interworking WLAN (I-WLAN) [24], [25].

Thus, while services offered through data cellular networks such as $3 \mathrm{G}$ and LTE have been a dominant technology, 802.11-based WLANs continue to exist as they can cater for a huge on-demand offloading on Telco networks and offer a higher throughput than cellular-based data networks.

\section{SETTING AND MethodS}

In undertaking this work, it was necessary a methodology in a way that could ensure the two simulation scenarios selected for this study, would be carried out in exactly the same way, and that there was consistency in the way the results were obtained from the simulated scenarios. The extent of its success is confirmed by a flowchart in Fig.2, which describes a sequence of steps on the basis of which the work was carried out.

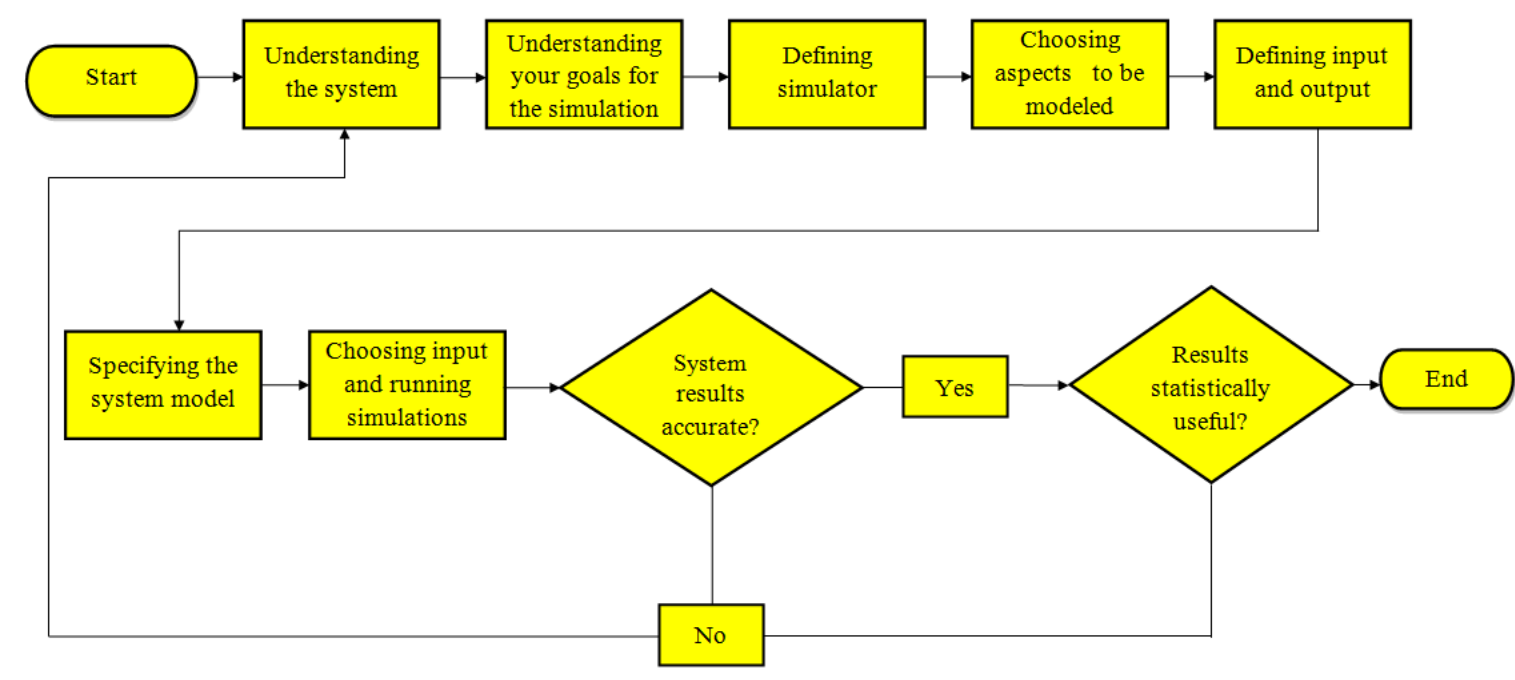

Fig.2. Flowchart [33]

The scenarios of interest were modeled with the aid of Opnet Modeler 17.5 [26] (in which its model implementations are mostly based on LTE release 8). A screenshot of the opnet implementation of the two scenarios is shown in Figure 3 and 4. In the analysis conducted for this study, two identical scenarios were created, one for WLAN and the other using femtocells.

\section{A. Simulation Assumptions}

The simulation was conducted through a survey of the initial configurations carried out in the OPNET Modeler. The simulation time was 1200 seconds, which is long enough for the environment to have a stable behavior and the test users to move around the network.

Several instances were run and simulations of scenarios with the same configuration were repeated. Time to elapse was the same and with same parameters but with different seeds. The configuration and parameterization of femtocell radios and WLAN are displayed in Table 4 and 5. With regard to mobility, it was assumed that all mobile users employ the random waypoint mobility model [27]. 
VoIP and videoconferencing applications were made use of to generate traffic on the network. These applications are used to represent the class of inelastic applications: that are real-time, interactive, sensitive to delay end-to-end, but able to tolerate packet loss. Today, the emergence of real-time applications requires more resources, and hence it is necessary to ensure rapid and reliable voice communication for a large number of users in the network. In Table 2 and 3 we list the most significant parameters that are used for configuring the application.

TABLE II. CONFIGURATION OF VoIP APPLICATION

\begin{tabular}{cc}
\hline Parameters & Values \\
\hline Silence Lengh (s)Data Rates & Exponentially distributed, mean 0.65 \\
Talk Spurt Length (s) & Exponentially distributed, mean 0.352 \\
Encoder Scheme & GSM FR \\
Voice Frames per Packet & 1 \\
Type of Service & Best effort (0) \\
\hline
\end{tabular}

TABLE III. CONFIGURATION OF VIDEO CONFERENCING APPLICATION

\begin{tabular}{cc}
\hline Parameters & Values \\
\hline Frame Interval Time Information & 10 frames/sec \\
Frame Size Information & $128 \times 120$ pixels \\
Type of Service & Best effort (0) \\
Voice Frames per Packet & 1 \\
Traffic Mix $(\%)$ & All Discrete \\
\hline
\end{tabular}

\section{B. Simulation Parameters of Femtocell}

In fig. 3 a typical indoor femtocell is shown, where different indoor users can connect to the Femtocell Acess Point (FAP) and use data and voice services. Femtocells use the same physical layer technology as cellular networks and are standardised since 3GPP release 8. More detail about the femtocell standardisation is provided in section IV of this paper.

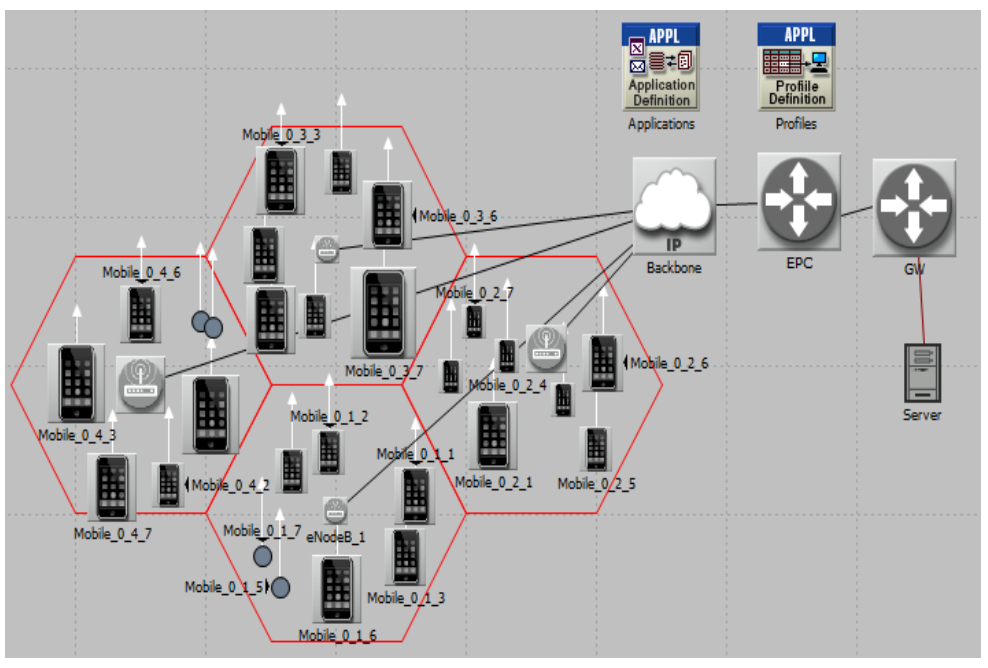

Fig.3. Scenario femtocell modeled in OPNET tool

The main parameters of configurations of femtocells radios used are in table IV. 
TABle IV. CONFiguration OF FEMtocells

\begin{tabular}{cc}
\hline Attributes & Settings \\
\hline EnodeB Tx Power & $23 \mathrm{dBm}$ \\
Bandwidth & $3 \mathrm{MHz}$ \\
Gain Antenna & $2 \mathrm{dBi}$ \\
Propagation Model & Indoor Environment \\
Duration of simulation & $1200 \mathrm{~s}$ \\
\hline
\end{tabular}

\section{Simulation Parameters of WLAN}

The IEEE $802.11 \mathrm{~b}$ has become the de facto standard for wireless networking technology among both small business and home users. The IEEE $802.11 \mathrm{~b}$ specifications allow for the theoretical transmission of approximately $11 \mathrm{Mbps}$ of raw data at indoor distances from several dozen to several hundred feet, and outdoor distances of several to tens of miles through an unlicensed use of the $2.4 \mathrm{GHz}$ wireless band. The $802.11 \mathrm{~b}$ choice was made considering the worst case used in practical experiment that are originated. Although the standard has been upgraded by others versions, a scenario which is thought to be close to the real worst case may be considered. The $802.11 \mathrm{~b}$ is the lowest cost amongst the standards and it is able to support the chosen applications.

\begin{tabular}{cc}
\multicolumn{2}{c}{ TABLE V. CONFIGURATION OF WLAN } \\
\hline Attributes & Settings \\
\hline Wlan Standard & $802.11 \mathrm{~b}$ \\
Transmit Power & $0.005 \mathrm{w}$ \\
Physical Characteristic & OFDM \\
Frequency & $2.5 \mathrm{GHz}$ \\
Data Rate & $11 \mathrm{Mpbs}$ \\
Roaming Capability & Enable \\
Duration of simulation & $1200 \mathrm{~s}$ \\
\hline
\end{tabular}

This network architecture is built around a Basic Service Set, which is actually a set of STAs that communicate with each other. When one access points is connected to wired network and a set of wireless stations it is referred to as a BSS. Basically, the Wlan scenario was composed with four wireless routers, each one with two WLAN interfaces; one of them serves as an access point for BSS 0, BSS 1, BSS 2, and BSS3 while the other interface makes up the WLAN-backbone. Every BSS was working as an independent wireless LAN.

The WLAN is connected via its AP to an office LAN connected through a central switch using 100BaseT (100Mbps) Ethernet wiring which emulates a real- life office environment. An IP gateway (i.e., an enterprise router) connects the LAN to an IP cloud used to represent the backbone Internet. The gateway connects to the office LAN using 100BaseT Ethernet wiring while the connection between the gateway and the IP cloud is effected with a Point-to-Point T3 serial link. 


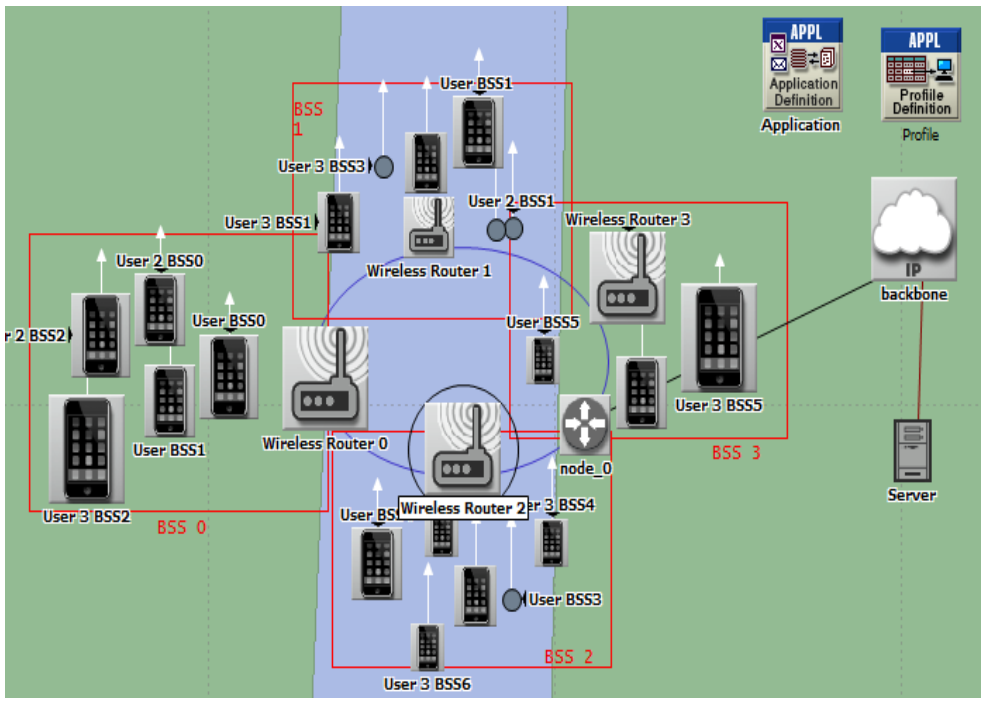

Fig.4. Scenario Wlan modeled in OPNET tool

\section{RESULTS AND DISCUSSION}

The results obtained show a comparison between a WLAN and femtocell environment. The purpose of this was to simulate two identical scenarios that represent current LTE mobile network deployments. The first scenario comprises typical LTE traffic found in indoor hotspots like bus stops, commercial streets, shopping centers, etc, within a femtocell coverage area. Scenario 2 involves the same configurations, but makes use of the Wifi routers.

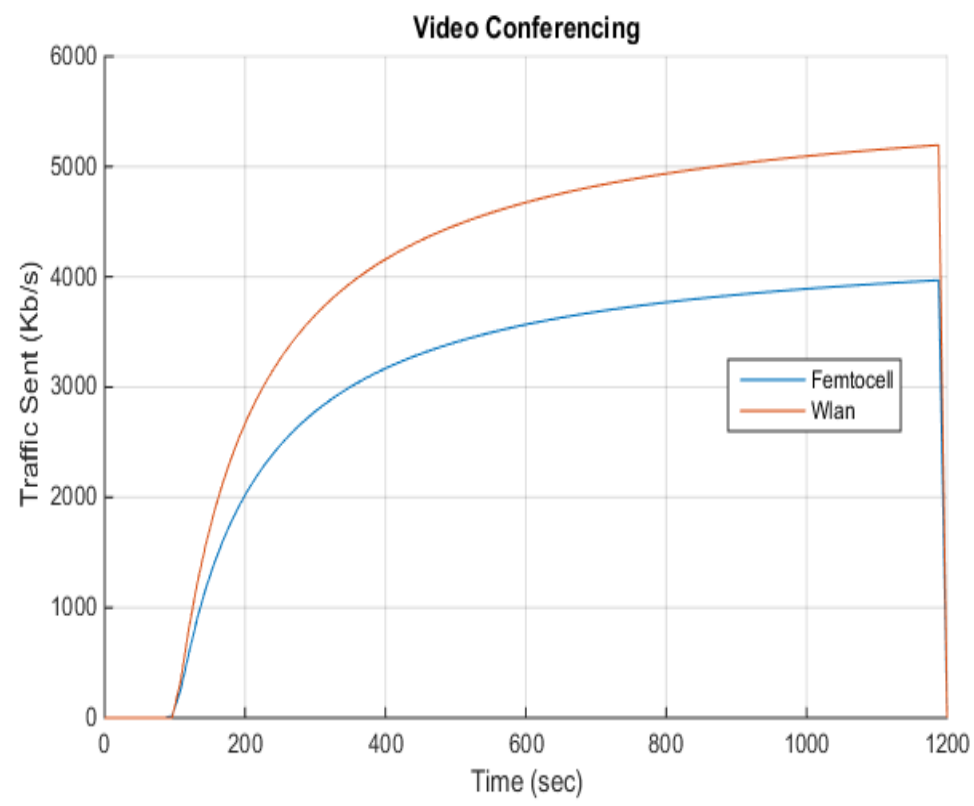

Fig.5. Video Conferencing - Traffic Sent

In Fig. 5 above, we observed global traffic that was sent around the network. It is worth noting that the traffic sent in both directions, starts at around 100 seconds and then remained stable, although the WLAN network achieved a better performance than the femtocells, and transmitted approximately 1 megabyte of data. 


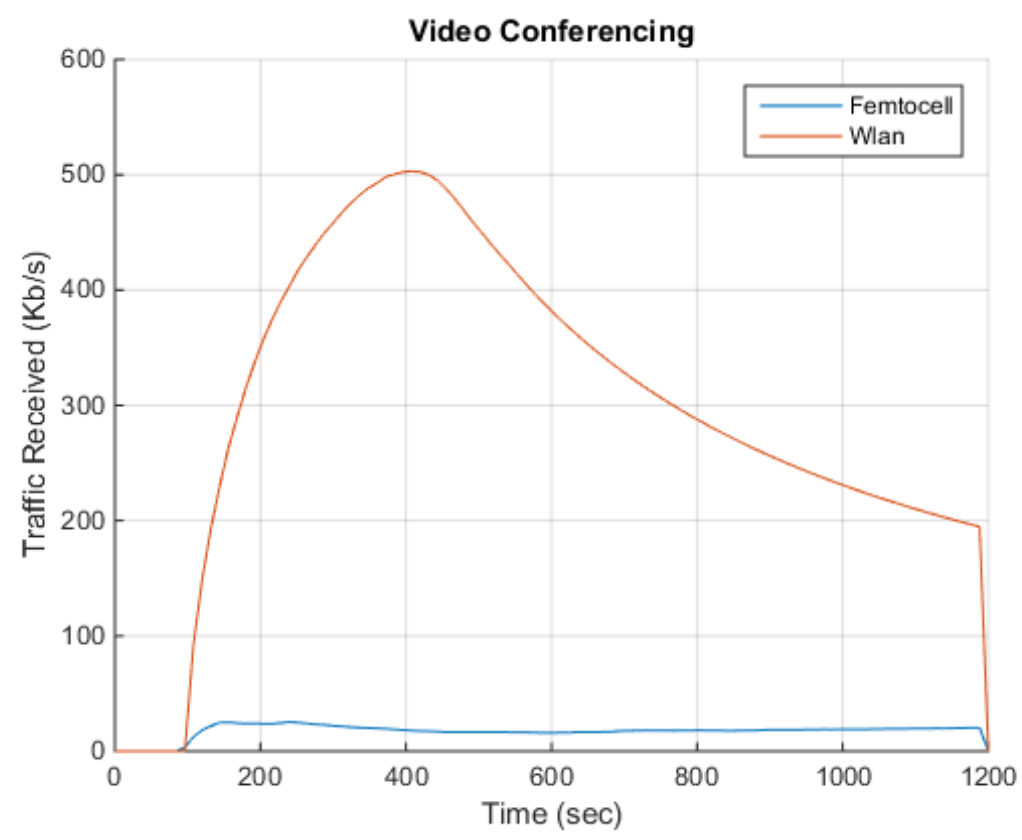

Fig.6. Video Conferencing - Traffic Received

Fig.6 shows the overall incoming traffic in the networks. It can be seen that the data received in the WLAN begins with a peak usage over time and remained for around $300 \mathrm{kbp} / \mathrm{s}$, and yet was much larger than the femtocell, where it remained stable for around $20 \mathrm{kbp} / \mathrm{s}$. A key factor in the femtocell environment, are the interferences. Femtocell deployments create a two-tier network, as a result of which interference can be either co-tier or cross-tier. When there is co-tier interference, a femtocell causes interference to a neighboring femtocell, which may be severe in the case of dense deployments. The cross-tier interference results when a femtocell causes interference to the downlink of a nearby macrocell user.

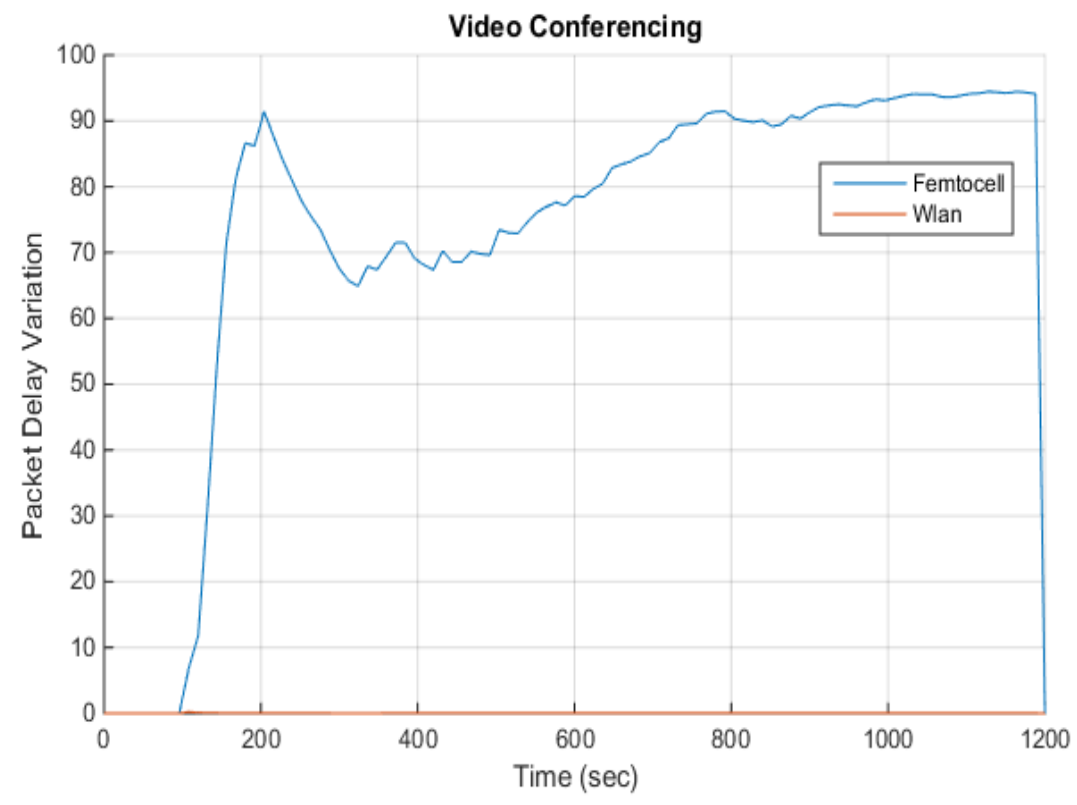

Fig.7. Video Conferencing - Packet Delay Variation

Delay is an essential metric to characterize the QoS of any network, especially for real time multimedia applications. Delay is defined as the time taken by the system for the data to reach the destination after it 
leaves the source. Fig. 7 shows the packet delay variation. There is clearly a big difference in behavior between the two scenarios. The femtocell network has around 70 to 90 packets, while WLAN obtained a negligible value during the whole simulation time. WLAN and femtocell work in different frequencies, but WLAN does not face problems with interference when compared with femtocells, thus as the interference do not occur in WLAN then delay metric is negligible and the same does not occur with femtocell.

The figures below show the results of the VoIP application. The VoIP applications generate packets at regular intervals, but after passing the intermediate network routers, the time intervals between the packages become completely irregular. Figures 8 and 9 show the global traffic that was sent and received respectively. The traffic from both networks has increased gradual growth.

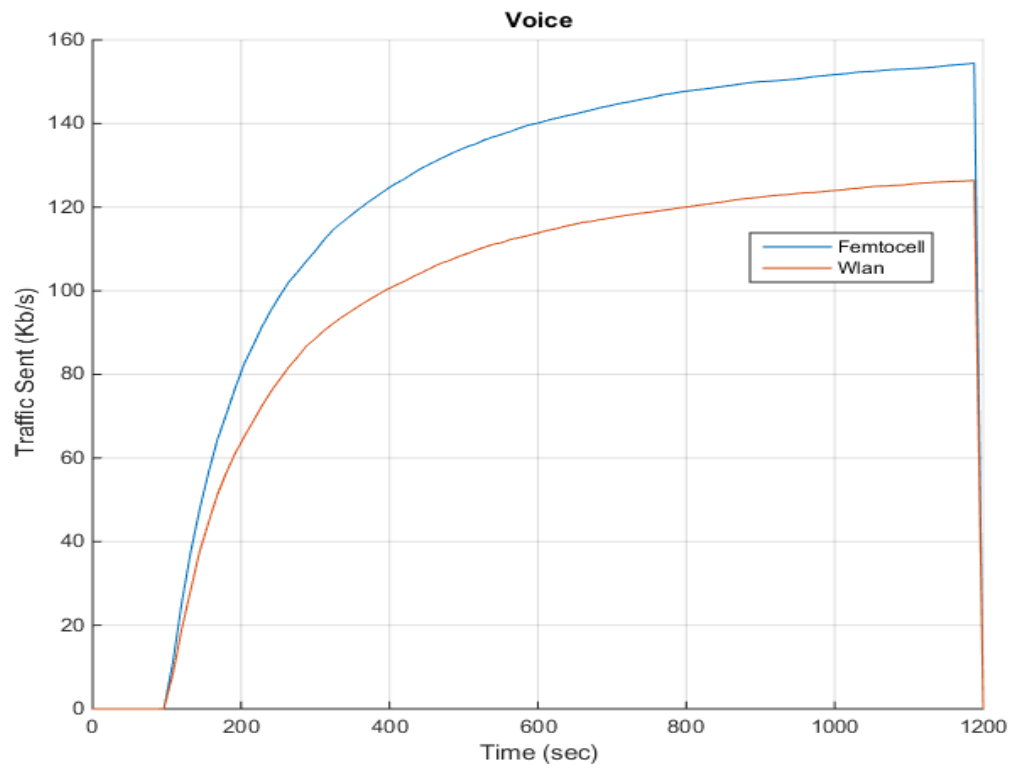

Fig.8. VoIP - Traffic Sent

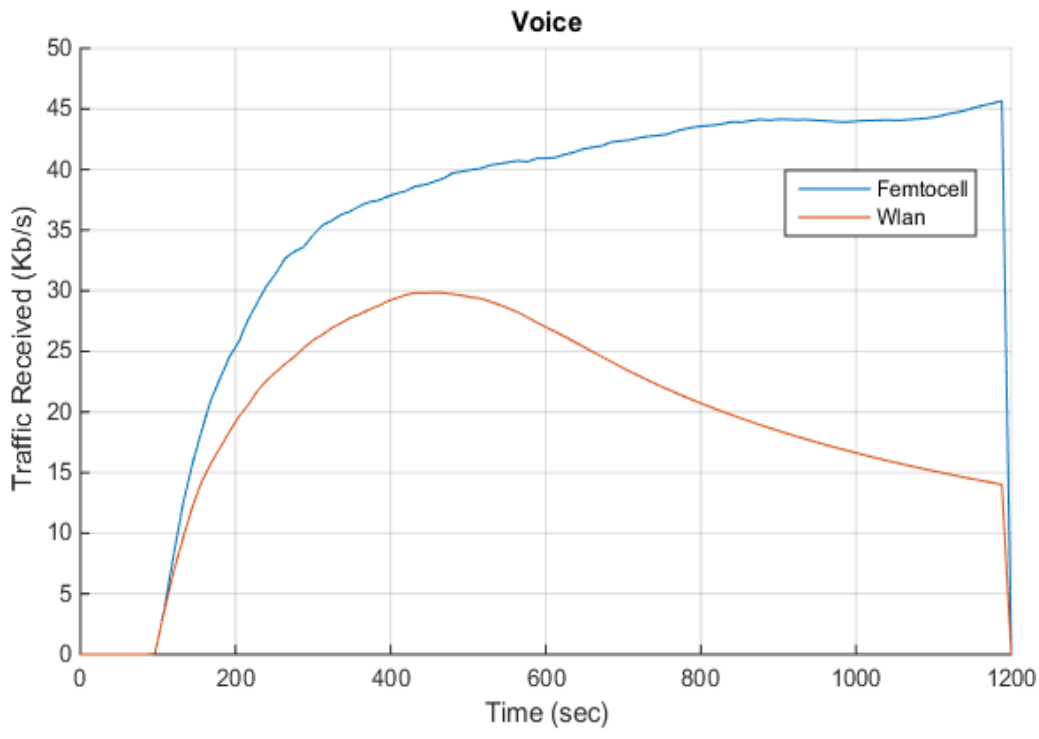

Fig.9. VoIP - Traffic Received 


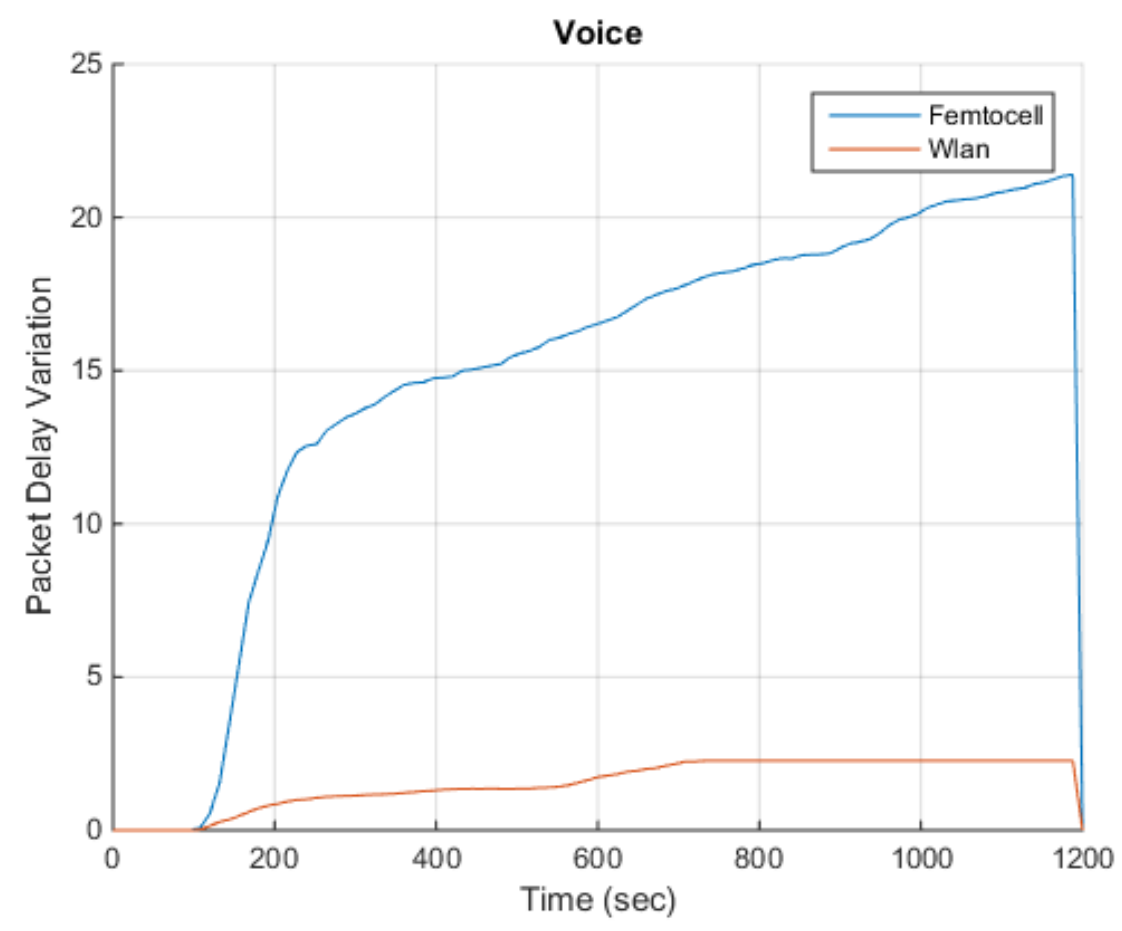

Fig.10. VoIP - Packet Delay Variation

The eye and ear are sensitive to delays in interactive conversation, but are perhaps even more sensitive to delay variations. However, they are less sensitive to bit errors, especially the ear, since one bit represents very little information. Fig. 10 shows the packet delay variation that is a measure of the difference in the Endto-End delay between packets in a flow, but ignores any packets that have been lost. It can be seen that for the WLAN scenario the delay does not exceed $3 \mathrm{~ms}$, while in the femtocell scenario, the value is growing exponentially. In addition to the fact that WLAN does not face problems with interference when compared with femtocells, the WLAN networks have a much greater range. 


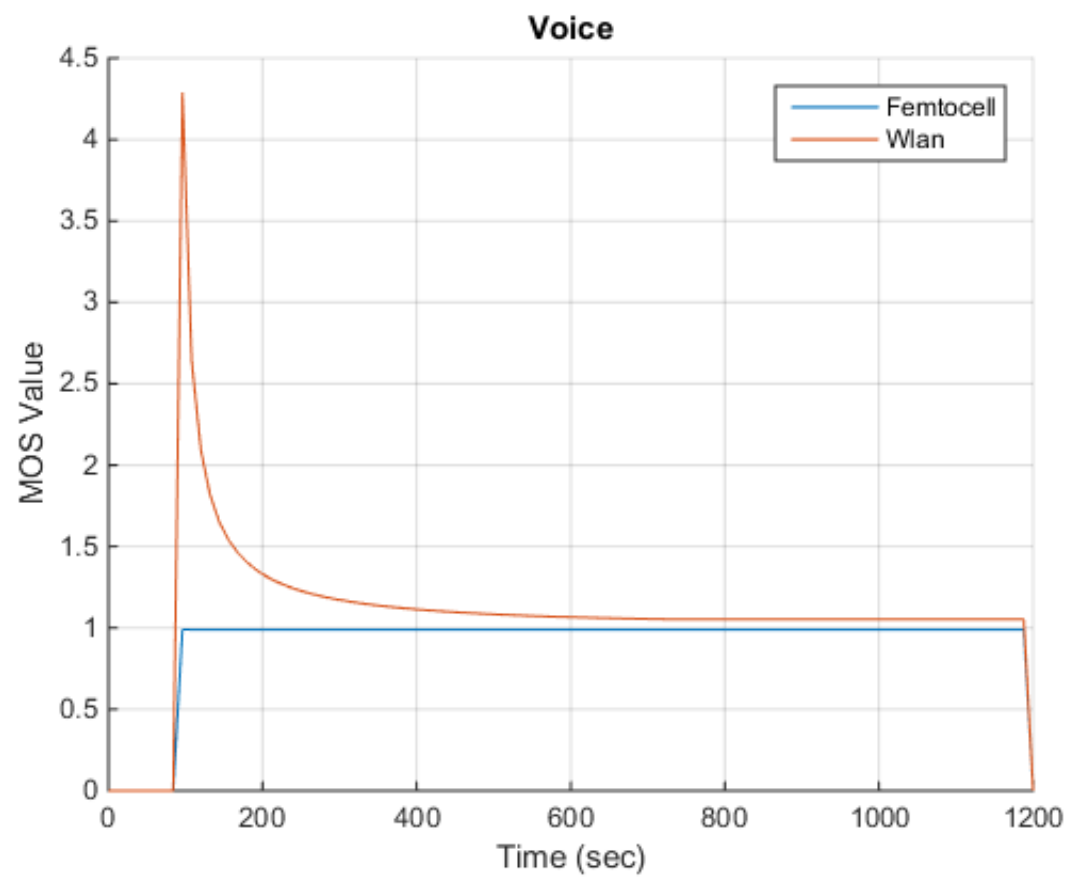

Fig.11. VoIP - MOS (Mean Opinion Score)

Fig. 11 shows the values of MOS obtained. The MOS is the mean average of the results from the users that tested the scenarios. Using a scale from 1 to 5 , where an average score equals or is greater than 4 , this is considered to provide toll-quality. The MOS achieved was considered poor, with both values below 2 . Although the WLAN initially started with a value of 4.5, both kept approximate values due to the high saturation of the network.

\section{CONCLUSIONS}

This paper outlines different issues associated with two competing technologies Femtocells and Wlan. Femtocells and Wlan both have the potential of providing next generation communications services. However, for the proposed scenarios the analysis allows us to infer that femtocells do not guarantee a better performance when compared with a wlan scenario.

The femtocell presented the worst performance in most of the results. To the best of our knowledge, it is known that interference influences PMOS and packet loss and they have been classified as the metrics that most degraded voip applications [28]-[29], which is demonstrated in the worst results of MOS and packet delay variation of the femtocell scenario.

For femtocells to become widely acceptable there are a number of challenges. Currently femtocells are being provided by operators, but due to the still low deployment numbers, the challenges are not yet apparent in practice. Femtocells would face many problems when the deployment is on a large scale and their density increases.

This paper has focused on extensive analysis until the capacity crunch of both technologies. From all that has been discussed, it is clear that Wlan networks can not be trashed, since worldwide there have been huge investment in infrastructure to open networks. 
According to [30], more than $70 \%$ of the population of the developing countries does not have access to Internet due to lack of infrastructure; furthermore, in countries like China, India and Brazil, with continental dimensions, the construction of a new telecommunications network, becomes costly and impractical, reinforcing the need for alternative lower-cost, deployment time and widely available. All this results prove the feasibility of implementing system using existing WLAN infrastructure or better it is possible to combine both.

From a technical point of view, as Wifi penetration increases, cellular (LTE-A) and Wifi networks should be able to handoff users seamlessly among them. The provisions are in 3GPP like access network discovery and selection function. However, there is still many proposals of improvements to use ANDSF standard [31], mainly because access selection may be done on different time scales, based on different parameters.

Future work includes expand this research with the study on perfomance-centric offload strategies for heterogeneous network based on non delayed traffic, AP-based strategy, 3GPP standardization usage according with [32]. We will propose an optimizer multiobjective for a hybrid access possibility with both technology working together take into considerations factors such as SNR and network traffic load, MOS or any parameters described in this work.

\section{REFERENCES}

[1] Haider, F.; Dianati, M.; Tafazolli, R., "A simulation based study of Mobile Femtocell assisted LTE networks,"Wireless Communications and Mobile Computing Conference (IWCMC), 2011 7th International, vol., no., pp.2198, 2203, 4-8 July 2011.

[2] Silva, K.; Silva, P.; Donza, A.; Frances, C.; Vijaykumar, N., "Analysis of Handover Based on The Use of Femtocells in LTE Networks" International Journal of Recent Development in Engineering and Technology(IJRDET), Volume 2, pp. 19-28, Issue 4, June 2014.

[3] Hasan, S.F.; Siddique, N.H.; Chakraborty, S., "Femtocell versus WiFi - A survey and comparison of architecture and performance,"Wireless Communication, Vehicular Technology, Information Theory and Aerospace \& Electronics Systems Technology, 2009. Wireless VITAE 2009. 1st International Conference on, vol., no., pp.916, 920, 17-20 May 2009.

[4] Li, Q.C. , Huaning Niu, Papathanassiou, A.T., Geng Wu. "5G Network Capacity: key elements and technologies", IEEE Vehicular Technology Magazine, 2014.

[5] MAIER M., and VERIKOUKIS C. "Inside Smart FiWi-HetNets and the Explosion of Mobile Data Traffic", International Conference on Transparent Optical Networks (ICTON), 2014.

[6] J. Zhang, G. D. L. Roche, and G. L. D. Roche, Femtocells: Technologies and Deployment. John Wiley and Sons, Ltd., 2010.

[7] Zahir, T.; Arshad, K.; Nakata, A.; Moessner, K., "Interference Management in Femtocells," Communications Surveys \& Tutorials, IEEE , vol.15, no.1, pp.293,311, First Quarter 2013.

[8] K. Lee, J. Lee, Y. Yi, I. Rhee and S. Chong, "Mobile Data Offloading: How Much Can WiFi Deliver?," Networking, IEEE/ACM Transactions on, vol.21, no.2, pp.536-550, April 2013

[9] S. Dimatteo, P. Hui, B. Han and V.O.K. Li, Cellular traffic offloading through WiFi networks. Proceedings of the 8th International Conference on Mobile Adhoc and Sensor Systems, pp. 192-201, October 2011.

[10] A. Balasubramanian, M. Ratul and V. Arun, Augmenting mobile 3G using WiFi. Proceedings of the 8th Annual International Conference on Mobile Systems, Applications and Services, San Francisco, CA., USA., pp. 209-222, June 2010

[11] Y. Li, Z. Lu and X. Wen, Adaptive Traffic Offloading Method Based on OWN in Overlay Networks with LTE and WLAN. Information Technology Journal, 13, pp. 2581-2587, 2014.

[12] Blankenship, Y.W., "Achieving high capacity with small cells in LTE-A," Communication, Control, and Computing (Allerton), 2012 50th Annual Allerton Conference on, vol.1, no.1, pp.1680,1687, 1-5 Oct. 2012

[13] Hinger, D.; Kalbande, D., "Review of mobile data offloading through Wifi," Circuits, Systems, Communication and Information Technology Applications (CSCITA), 2014 International Conference on, vol., no., pp.425,429, 4-5 April 2014.

[14] Danish Aziz, Rolf Sigle, "Improvement of LTE Handover Performance through Interference Coordination”, IEEE 69th Vehicular Technology Conference, 2009.

[15] 3GPP TS 136.300 V8.12.0, Evolved Universal Terrestrial Radio Access (E-UTRA) and Evolved Universal Terrestrial Radio Access Network (E-UTRAN). Overall Description; Stage 2. (Release 8) 2010.

[16] V. Chandrasekhar, J. Andrews, and A. Gatherer, "Femtocell Networks: A Survey," Communications Magazine, IEEE, vol. 46, no. 9, pp. 59 -67, September 2008.

[17] T. Zahir, K. Arshad, A. Nakata, and K. Moessner, "Interference management in femtocells," Communications Surveys \& Tutorials, IEEE, vol. 15,no. I, pp. 293-311, 2013.

[18] K. Dimou, M. Wang, Y. Yang, M. Kazmi, A. Larmo, J. Pettersson, W. Muller, and Y. Timner, "Handover within 3GPP LTE: Design Principles and Performance," in Vehicular Technology Conference Fall (VTC 2009-Fall), 2009 IEEE 70th, September 2009, pp. 1-5 
[19] Prasad N., Prasad A., 802.11 WLANs and IP Networking: Security, QoS, and Mobility, Artech House Publishers, 2005.

[20] Cisco, "The Future of Hotspots: Making Wifi as Secure and Easy to Use as Cellular", white paper, 2013.

[21] China Mobile WiFi services. http://tinyurl.com/m7lj366.

[22] KDDI WiFi services. http://tinyurl.com/mzpelfv.

[23] AT\&T WiFi services. http://www.att.com/gen/general?pid=5949.

[24] 3GPP TS 23.234 "3GPP system to Wireless Local Area Network (WLAN) interworking; System description".

[25] 3GPP TS 24.234 "3GPP System to Wireless Local Area Network (WLAN) interworking; WLAN User Equipment (WLAN UE) to network protocols; Stage 3".

[26] Opnet Modeler. [Online]. Available: http://www.riverbed.com/products/performance-management-control/opnet.html

[27] T. Camp et al., A Survey of Mobility Models for Ad Hoc Network Research, Wireless Communications and Mobile Computing, vol. 2, no. 5, pp. 483-502, 2002.

[28] Zahir, T. et al. Interference Management in Femtocells. IEEE, Communications Surveys and Tutorials. 2013.

[29] Rodrigues, J. C.; Fraiha, S. G. C.; Araújo, J. P.; Gomes, H. S.; Francês,C. R. L.; Cavalcante, G. P. S. "Empirical Study of the QoS Parameters Behavior of a VoIP Application in Wifi Networks", International Microwave and Optoelectronics Conference, Belém-Pará, IMOC 2009.

[30] IWS - Internet World Status (2014). Usage and Population Statistics, 2014. Available at http://www.internetworldstats.com/stats.htm.

[31] 3GPP TS 24.312: Access Network Discovery and Selection Function (ANDSF) Management Object (MO) (Rel. 10), June 2011.

[32] Rebecchi, F.; Dias de Amorim, M.; Conan, V.; Passarella, A.; Bruno, R.; Conti, M., "Data Offloading Techniques in Cellular Networks: A Survey," in Communications Surveys \& Tutorials, IEEE, vol.17, no.2, pp.580-603, Secondquarter 2015.

[33] SILVA, K. C.; FRANCES, C. R. L. Analysis of Handover based on the use of Femtocells in LTE Networks. 1. ed. Saarbrücken: LAP LAMBERT Academic Publishing, 2015. 88p. [Online]. Available: https://www.amazon.com.br/Analysis-Handover-Based-Femtocells-Networks. 